\title{
Solution of a 3-D Multilayered Poroelastic Medium Subjected to Moving Load with TRM Method
}

\author{
Sheng Guo-jun Huang Qian \& XU Man-qing \\ Nanchang Institute of Technology, Nanchang, Jianxi, 330029, China
}

KEYWORD: wave localization; periodic viaduct; Lyapunov exponent; disordered

ABSTRACT: The focus of this paper is to develop a transmission and reflection matrices (TRM) method for 3-D layered poroelastic half-space subjected to moving load. Applying the triple Fourier transformation, the general solution for the displacements, stress and pore pressure are derived from the governing equations of Biot's theory. considering the continuity conditions at layer interface and the boundary conditions at the top surface as well as moving load, the transmission and reflection matrices(TRM) method for the layered porous medium is developed .Some numerical example are given in this paper.

\section{INTRODUCTION}

In recent years many high-speed ground transports have been established on the fluid-fill situated soil, railroad track move over soft soil surface with high velocity must radiated waves propagate in the subsoil and strongly affect the dynamic behavior of the track. In the developing a mathematical model of the track, this phenomenon has to be taken into account by including a 3-D model of satuated subsoil. However the most existing published works on this subject mainly considered that subsoil is modeled as a linear elastic or viscoelastic homogeneous or layered half space. the twodimensional problem of a line load moving with uniform subsonic velocity over the surface of uniform elastic half space was first considered by Sneddon[1,2] in 1951.Cole and Huth[3] considered the same problem for a normal line load and obtained solutions for the subsonic, transonic and supersonic case. Metrikine and Vrouwenvelder[4] proposed a model for the surface ground vibration due to a moving train in a tunnel. Grundmann et al. [5] studied the response of a layered half-space subjected to a single moving periodic load as well as a simplified trainload. The three-dimension problem of the steady-state motion of a point load in an unbounded body was considered by Eason et al. [6].Mandel and Avramesco[7] investigated the displacement of an elastic half-space subjected to a point load moving with constant velocity on its surface. Luco and Barros[8] studied the steady-state response of a multi-layered viscoelastic half-space subjected to a buried or surface point moving with an arbitrary constant velocity in a fixed horizontal direction. Hung and Yang[9] studied the elastic waves in viso-elastic half-space generated by various vehicle loads..

The objective of the present paper is directed to the study of the steady-state response of the threedimensional multi-layered porouselastic half space subject to a surface moving load with an arbitrary constant velocity. The Biot's dynamic equations are solved by Fourier transform techniques. the general solutions for the displacements, stresses and pore pressure et al. are obtained .Using the general solutions for displacements, stresses and pore pressure and the continuity conditions at the layer interfaces as well as the boundary conditions, the transmission and reflection matrices (TRM) of the porous layers surface moving point with an arbitrary constant velocity are derived. The final response in time domain is obtained from the frequency-wave-number solution by use of discrete Fast Fourier invert transformation. When reduced to some special cases, our fundamental solutions agree very well with known results by comparisons with published solution available for simple limiting cases (elastic half-spce). In order to demonstrate the method, two time domain numerical examples and corresponding analysis are presented in the paper. 


\section{GOVERNING EQUATIONS AND THE GENERAL SOLUTIONS}

The constitutive equations for homogeneous porous medium have the form [10],[11]

$$
\begin{gathered}
\mu u_{i, j j}+\left(\lambda+\alpha^{2} M+\mu\right) u_{j, i i}-\alpha M w_{j, j i} \\
=\left(\rho+\rho_{f}\right) \\
M\left(u_{j, j i}+w_{j, j i}\right)=\left(\rho_{f}+m \alpha_{l}\right.
\end{gathered}
$$

where over dots denote the derivative with respect to the time parameter $t ; u_{i}$ and $w_{i}(i=1,2,3)$ are the displacement of solid matrix and the fluid displacement relative to the solid matrix, respectively; $\lambda$ and $\mu$ are Lame constants; $\alpha$ and $\mathrm{M}$ are Biot's parameters accounting for compressibility of the twophased material; $\rho$ and $\rho_{\mathrm{f}}$ are mass densities of the bulk material and the pore fluid, respectively; $m$ is a density-like parameter that depends on $\rho_{\mathrm{f}}$ and the geometry of the pores; $b$ is a parameter accounting for the internal friction due to the relative motion between the solid matrix and the pore fluid. The parameter $b$ is equal to the ratio between the fluid viscosity and the intrinsic permeability of the medium. If internal friction is neglected then $b=0$.

The constitutive relations can be expressed as

$$
\begin{aligned}
\sigma_{i j} & =\lambda e \delta_{i j}+\mu\left(u_{i, j}+u_{j, i}\right)-\alpha \delta_{i j} p \\
p & =-\alpha M \theta+M e
\end{aligned}
$$

Where $\theta=\mathrm{u}_{\mathrm{i}, \mathrm{j}}$ and $\mathrm{e}=\mathrm{w}_{\mathrm{i}, \mathrm{j}}$ is solid and fluid strain; $\sigma_{\mathrm{i}, \mathrm{j}}$ is the total stress component of bulk material; $\mathrm{p}$ is the pore fluid pressure.

To solve the problem the equation of motion is Fourier transformed with respect to the timet $\rightarrow \omega$, the Fourier transformed defined by:

$$
\hat{f}(x, y, \omega)=\int_{-\infty}^{\infty} f(x, y, t) e^{-i \omega t} d t
$$

In this paper, the symbol ${ }^{\wedge}$ is used denote $\mathrm{t} \rightarrow \omega$ Fourier transformed. Then one can show that application of $\mathrm{t} \rightarrow \omega$ Fourier integral transforms to Esq. (1) and (2) leads to

$$
\begin{aligned}
& \mu \nabla^{2} \hat{u}_{x}+\left(\lambda+\alpha^{2} M+\mu\right) \frac{\partial \hat{\theta}}{\partial x}+\omega^{2}\left(\rho-\rho_{f} \vartheta\right) \hat{u}_{x}-(\alpha-\vartheta) \frac{\partial \hat{P}}{\partial x}=0 \\
& \mu \nabla^{2} \hat{u}_{y}+\left(\lambda+\alpha^{2} M+\mu\right) \frac{\partial \hat{\theta}}{\partial y}+\omega^{2}\left(\rho-\rho_{f} \vartheta\right) \hat{u}_{y}-(\alpha-\vartheta) \frac{\partial \hat{P}}{\partial y}=0 \\
& \mu \nabla^{2} \hat{u}_{z}+\left(\lambda+\alpha^{2} M+\mu\right) \frac{\partial \hat{\theta}}{\partial z}+\omega^{2}\left(\rho-\rho_{f} \vartheta\right) \hat{u}_{z}-(\alpha-\vartheta) \frac{\partial \hat{P}}{\partial z}=0 \\
& \nabla^{2} \hat{P}+\frac{\rho_{f} \omega^{2}}{\vartheta M} \hat{P}+\rho_{f} \omega^{2}(\alpha-\vartheta) \frac{\hat{\theta}}{\vartheta}=0
\end{aligned}
$$

where

$\nabla^{2}$ is the Laplacian operator defined by

$$
\vartheta=\frac{\rho_{f} \omega^{2}}{m \omega^{2}-i b \omega}
$$

$$
\nabla^{2}=\partial^{2} / \partial x^{2}+\partial^{2} / \partial y^{2}+\partial^{2} / \partial z^{2}
$$

From Esq. (2-4) we obtain:

$$
\left(\lambda+\alpha^{2} M+2 \mu\right) \nabla^{2} \hat{\theta}+\omega^{2}\left(\rho-\rho_{f} \vartheta\right) \hat{\theta}-(\alpha-\vartheta) \nabla^{2} \hat{P}=0(7)
$$

From Esq. (7), the following equation can be obtained:

Substituting Esq. (7) into Esq. (8) yields:

$$
\hat{\theta}=-\frac{\nabla^{2} \hat{p} \vartheta}{\rho \omega(\alpha-\vartheta)}-\frac{\hat{p}}{(\alpha-\vartheta) M}
$$

$$
\nabla^{4} \hat{p}+\beta_{1} \nabla^{2} \hat{p}+\beta_{2} \hat{p}=0
$$

where

$$
\beta_{1}=\frac{\left(m \omega^{2}-i b \omega\right)\left(\lambda+\alpha^{2} M+2 \mu\right)+\rho \omega^{2} M-2 \alpha M \rho_{f} \omega^{2}}{(\lambda+2 \mu) M}
$$




$$
\beta_{2}=\frac{\left(m \omega^{2}-i b \omega\right) \rho \omega^{2} M-\rho_{f}{ }^{2} \omega^{4}}{(\lambda+2 \mu) M}
$$

The Double Fourier integral transform of function $f(x, y, z)$ with respect to the two horizontal coordinates $\mathrm{x} \rightarrow \xi, \mathrm{y} \rightarrow \eta$ are defined by:

$$
\tilde{\bar{f}}(\xi, \eta, z)=\int_{-\infty-\infty}^{\infty} \int_{-\infty}^{\infty} f(x, y, t) e^{-i \xi \xi} e^{-i \eta y} d x d y
$$

The symbol -, and are used denote $\mathrm{x} \rightarrow \xi, \mathrm{y} \rightarrow \eta$ Double Fourier transformed. The inverse relationship is given by

$$
f(x, y, z)=\frac{1}{4 \pi} \int_{-\infty-\infty}^{\infty} \int_{-\infty}^{\infty} \tilde{\tilde{f}}(\xi, \omega, z) e^{i \xi x} e^{i \eta y} d \xi d \eta
$$

Thus, application of the Double Fourier integral transformations to Esq. (9) leads to

$$
\hat{\bar{p}}=A e^{-r_{1} z}+B e^{-r_{2} z}+C e^{-r_{1} z}+D e^{-r_{2} z}
$$

Where

$$
r_{i}=\sqrt{\xi^{2}+\eta^{2}-L_{i}^{2}} \quad \mathrm{i}-1,2
$$

Note that the radicals $r_{i}(i=1,2)$ are selected such that $\operatorname{Re}\left(r_{i}\right) \geq 0$. In addition,

$$
\begin{aligned}
& L_{1}^{2}=\frac{1}{2}\left(\beta_{1}-\sqrt{\beta_{1}{ }^{2}-4 \beta_{2}}\right) \\
& L_{2}{ }^{2}=\frac{1}{2}\left(\beta_{1}+\sqrt{\beta_{1}{ }^{2}-4 \beta_{2}}\right)
\end{aligned}
$$

where $\mathrm{L}_{1}$ and $\mathrm{L}_{2}$ are the dimensionless complex wave numbers associated with the dilatational wave of the first kind (fast wave), and the dilatational wave of the second kind (slow wave), respectively. Substituting $\hat{\bar{p}}(\xi, \eta, z, \omega)$ from Esq. (13) into the transformed form of Esq. (8) yields:

where

$$
\hat{\tilde{\theta}}=\chi_{1} A e^{-r_{1} z}+\chi_{2} B e^{-r_{2} z}+\chi_{1} C e^{r_{1} z}+\chi_{2} D e^{r_{2} z}
$$

$$
\chi_{i}=\frac{\vartheta M L_{i}^{2}-\rho_{f} \omega^{2}}{\rho_{f} \omega^{2}(\alpha-\vartheta) M} \quad \mathrm{i}=1,2
$$

Substituting $\hat{\tilde{\bar{p}}}(\xi, \eta, z, \omega)$ from Esq. (13) and $\hat{\tilde{\tilde{\theta}}}(\xi, \eta, z, \omega)$ from Esq. (16) into the transformed form of Esq. (4) and (5) yields:

Where

$$
\begin{aligned}
\hat{\hat{u}}_{z}^{\hat{o}}=r_{1} & a_{1} A e^{-r_{1} z}+r_{2} a_{2} B e^{-r_{2} z}-r_{1} a_{1} C e^{r_{1} z}-r_{2} a_{2} D e^{r_{2} z} \\
& +E e^{-r_{3} z}+F e^{r_{r_{3}} z} \\
\hat{\hat{u}} / 0= & -\dot{m}\left(a_{1} A e^{-r_{1} z}+a_{2} B e^{-r_{2} z}+a_{1} C e^{r_{1} z}+a_{2} D e^{r_{2} z}\right) \\
& +G e^{-r_{3} z}+H e^{r_{3} z}
\end{aligned}
$$

$$
\begin{aligned}
& a_{i}=\frac{\lambda\left(\chi_{i}+\mu\right)-\alpha+\vartheta}{\left(S^{2}-L_{i}^{2}\right) \mu} \quad i=1,2 \\
& r_{3}=\sqrt{\xi^{2}+\eta^{2}-S^{2}}
\end{aligned}
$$

Note that the radical $r_{3}$ is selected such that $\operatorname{Re}\left(r_{3}\right) \geq 0$ In addition,

$$
S^{2}=\left(-\rho_{f} \vartheta+\rho\right) \omega^{2} / \mu
$$

where $S$ is the dimensionless complex wave number associated with the rotational wave. Making use of the transformed form of the dilatation of the solid

$$
\hat{\tilde{\bar{\theta}}}=i \xi u_{x}+i \eta u_{y}+\partial u_{z} / \partial z
$$

$\mathrm{u}_{\mathrm{x}}$ can be obtained

$$
\begin{gathered}
i \xi \hat{u_{x}^{o}}=g_{1}\left(A e^{-r_{1} z}+C e^{r_{1} z}\right)+g_{2}\left(B e^{-r_{2} z}+D e^{r_{2} z}\right) \\
+r_{3}\left(E e^{-r_{3} z}-F e^{r_{3} z}\right)-\dot{m}\left(G e^{-r_{3} z}+H e^{r_{3} z}\right)
\end{gathered}
$$

By virtue of Esq. (2) the three total stress components on a plane perpendicular to the $\mathrm{z}$-axis can be obtained 


$$
\begin{aligned}
\hat{\partial} \%= & g_{3}\left(A e^{-r_{1} z}+C e^{r_{z} z}\right)+g_{4}\left(B e^{-r_{2} z}+D e^{r_{2} z}\right) \\
& +2 G r_{3}\left(E e^{-r_{3} z}-F e^{r_{3} z}\right) \\
i \xi \hat{\tau} \%= & g_{5}\left(A e^{-r_{1} z}-C e^{r_{1} z}\right)+g_{6}\left(B e^{-r_{2} z}-D e^{r_{2} z}\right) \\
- & \left(\xi^{2}+r_{3}^{2}\right)\left(E e^{-r_{3} z}+F e^{r_{3} z}\right)-i r_{3} \eta\left(H e^{r_{3} z}-G e^{-r_{r^{2} z}}\right)
\end{aligned}
$$

where

$$
\begin{aligned}
& \left.g_{i}=\chi_{i}+a_{i}\left(r_{i}^{2}-\eta^{2}\right)\right) \quad \\
& g_{i}=\lambda\left(\chi_{i}-2 \mu a_{i} r_{i}^{2}\right) \quad i=3,4 \\
& g_{i}=-r_{i}\left(\chi_{i}+a_{i}\left(r_{i}^{2}+\xi^{2}-\eta\right)\right) \quad i=5,6
\end{aligned}
$$

Using the continuity condition and the radiation condition, the generalized transmission matrices and reflection matrices which can be used to evaluate the up-going and down-going wave vectors at the $\mathrm{j}$-th porous layer below the source can also be obtained. The up-going and down-going wave vectors in $\mathrm{j}$-th layer have the form

Where

$$
\begin{aligned}
& W_{d}{ }^{j}\left(\xi, \eta, z_{j-1}, \omega\right)=\stackrel{g}{T}_{d e}{ }^{j-1} \stackrel{g}{T_{d e}} \quad \ldots \stackrel{g}{T}_{d e}{ }^{2} \stackrel{g}{T}_{d}{ }^{1} \\
& \times{\stackrel{g}{W_{d}}}^{1}\left(\xi, \eta, z_{1}, \omega\right) \\
& W_{u}{ }^{j}\left(\xi, \eta, z_{j}, \omega\right)=\stackrel{g}{R}_{d}{ }^{j} W_{d}{ }^{j}\left(\xi, \eta, z_{j}, \omega\right) \\
& j=2,3 \ldots \ldots \ldots . . N+1
\end{aligned}
$$

$$
\begin{gathered}
R_{d}^{g^{j}}=R_{d}{ }^{j}+T_{u e}{ }^{j} R_{d e}{ }^{j+1} T_{d}^{g^{j}} \quad j=1,2,3 \ldots \ldots . . N \\
T_{d e}^{g^{j}}=\left(I-R_{u e}{ }^{j} R_{d e}^{g^{j+1}}\right)^{-1} T_{d e}{ }^{j}
\end{gathered}
$$

In terms of the free surface boundary conditions Esq. (3-7a-d), the following relation is obtained

$$
\begin{aligned}
S_{d}{ }^{1} W_{d}{ }^{1}\left(\xi, \eta, z_{0}, \omega\right) & +S_{u}{ }^{1}(\xi, \eta, \omega) W_{u}{ }^{1}\left(\xi, \eta, z_{0}, \omega\right) \\
= & \hat{\tilde{F}}(\xi, \eta, \omega) \\
W_{d}{ }^{1}\left(\xi, \eta, z_{0}, \omega\right)= & \left(S_{d}^{1}+S_{u e}{ }^{1} R_{d e}^{g^{1}}\right)^{-1} \hat{\tilde{F}}(\xi, \eta, \omega)
\end{aligned}
$$

All the transmission and reflection matrices used to calculate the wave vectors of $j$-th layer from the source layer vectors have been obtained now. After the wave vectors in the first layer are determined, it is rather straightforward to obtain all the wave vectors in arbitrary layer.

\section{NUMERICAL RESULTS AND DISCUSSIONS}

The capability of the method to calculated the response of a multi-layered half space to moving point load illustrate by the case shown in Fig1, the medium consists of a two layered system will be considered. The upper is characterized by the Shear modulus of soil $\mu_{1}=$, thickness $h 1=2.0 \mathrm{~m}$, the lower half space are $\mu 2=$, the other parameters are equate as the first example the point load moves along the positive $x$-direction over the surface with velocity $c=70 \mathrm{~m} / \mathrm{s}$, the observer is located in the third layer at $\mathrm{A}(\mathrm{x}, \mathrm{y}, \mathrm{z})=(0,0,1.0 \mathrm{~m})$, the displacement components are normalizes according to $\mathrm{u}^{*}=\left(\mu \mathrm{z}_{\mathrm{A}} / \mathrm{F}_{\mathrm{z}}\right) \mathrm{u}_{\mathrm{z}}$ where $\mu=2.0 \mathrm{e}^{9} \mathrm{~N} / \mathrm{m}^{2}$.for comparison, the result plotted in the figures 2 with dash line. the corresponded elastic solution is compared with the solution of Barro[12].also plotted in the figures. It also notice that the multi-layered case exhibits higher frequencies and larger amplitudes than the response obtained for a uniform half space with the properties of the underlying half space. 


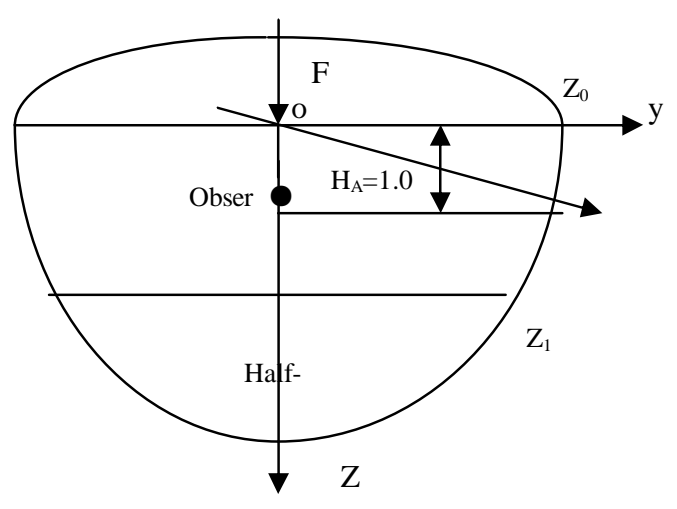

Fig.1 Two layered soil model

In the preceding examples, the present approach has been demonstrated to be capable of computing the homogeneous or layered medium by a moving load with subsonic and supersonic case.

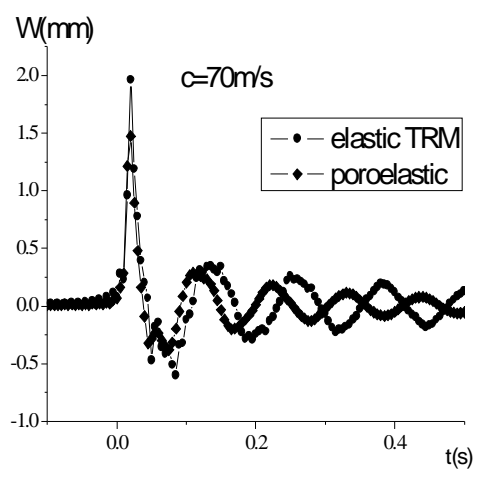

Fig.2 normalized vertical displacement $u^{*}$

\section{CONCLUSIONS}

Through above analysis and calculation the following conclusion can be drawn. Two-dimensional Fourier transformations applied to the time/ frequency and to the space/wave number domains lead to simple ordinary differential equations which can be solved analytically. A procedure to obtain the steady-state displacements and stresses within a multilayered poroelastic half-space with variable stiffness in the vertical generated by a surface moving point load in a horizontal straight line with constant speed has been presented. The validity and accuracy of the proposed methodology and associated computer program have been investigated by comparison with analytical and numerical result available for simple limiting case. Several features of response of a uniform poroelastic half space to surface load moving with constant subsonic, transonic and supersonic velocity have been presented. Some examples are given to illustrate typical features of the approach for a layered half space.

The fundamental solution for a moving load problem is of great importance for civil engineering, seismology and earthquake engineering. For example, grounding vibrations by surface traffic.

\section{ACKNOWLEDGMENTS}

The project is supported by the National Natural Science Foundation of China with grant number No. 51269021, 51569016. Also, the research is supported by the Jiangxi province science and technology plan projects(No: 20133ACB20006, 20141BBG70088). Moreover, the financial support from Science and technology project of Jiangxi Provincial Education Department Number GJJ14755 is greatly appreciated. 


\section{REFERENCES}

[1] Sneddon LN Fourier Transforms[M]. McGraw-Hill Book Company,1951, New York.

[2] Sneddon LN The stress produced by a pulse of pressure moving along the surface of a semiinfinite soil[J]. Rend.Cir.Mater.Palermo 1952, 2,57-62.

[3] Cole J, Huth J Stress produced in a half-space by moving loads[J]. J.Appl.Mech. 1958, 25,433436.

[4] Metrikine AV, Vrouwenvelder ACWM Surface ground vibration due to a moving train in a tunnel: two-dimensional model[J]. J Sound Vib 2000, 234(1):43-66.

[5] Grundmann H, Lieb M, Trommer E. The response of a layered halfspace to traffic loads moving along its surface[J]. Arch Appl Mech 1999, 69(1):55-67.

[6] Eason G, Fulton J, Sneddon IN Generation of waves in an infinite elastic solid by variable body forces[J]. Phi. Trans. Roy. Soc. A, 1956, 248,575-607.

[7]J.Mandel, Avramesco A Deplacements produits par une charge mobile a la surface dune semiespace elastique[J]. Comptes Rendus des Seances de L'Academie des Sciences, 1961, $252,3730-7735$.

[8] Debarros FCP, Luco JE Response of a layered viscoelastic half-space to a moving point load[J]. Wave Motion 11994, 9:189- 210

[9] Hung HH, Yang YB Elastic waves in visco-elastic half space generated by various vehicle loads[J]. Soil Dyn Earthquake Engng, 2001, 21:1-17.

[10] Biot MA Mechanics of deformation and acoustic propagation in porous media[J]. J. Appl. Phys. 1962, 33:1482-1498

[11] Plona $\mathbf{J}$ Observation of a second bulk compressional wave in a porous medium at ultrasonic frequency[J]. Appl. Phys. Lett. 1980, 36:259-261.

[12] Barros FCP, Luco JE Moving Green's Functions for a layered viscoelastic Half space[R]. 1992, Report of the Dept. of Appl. Mech. Engng. Sci, unit of California, san Diego,CA. 